\title{
Integrated biomarker discovery: combining heterogeneous data
}

\author{
"The most valuable biomarkers may well be integrated sets, best discovered through the systematic application of \\ multianalyte assays from a variety of instrument platforms, spanning several levels of biological processes."
}

Keywords: databases $₫$ data integration $\approx$ data mining $\|$ data warehouse $\approx$ integrated biomarkers $\approx$ OLAP $\approx$ systems biology

As a research community, we are seeing an increase in the number of analytes that can be measured in a single assay, and an increase in the number of studies that perform multiple assays on the same samples [1]. To fully exploit the resulting data, we need to combine heterogeneous data from diverse assays, associated sampledescriptive data, and clinical and demographic data into a cohesive whole. The discipline of data warehousing offers established tools and techniques for combining heterogeneous data. Recent advances in computing technology make data warehousing cheaper and faster. Furthermore, combined data provides a more detailed, comprehensive and, presumably, more accurate picture of the biological processes under investigation. In addition, a cohesive database provides us with a common vocabulary for discussing multiple assays and allows us to use a common tool kit to interrogate data from all assays, regardless of instrument platform. Such a database will greatly increase our ability to discover integrated biomarkers. In this work, we define an integrated biomarker as a set of characteristics that can be objectively measured and evaluated as an indicator of normal biologic processes, pathogenic processes or responses to a therapeutic intervention [2].

Our ability to measure multiple analytes in biological assays is ever increasing. For example, we can readily measure over 50 analytes in plasma or serum using Luminex ${ }^{\circledR}$-style bead arrays. Whole genome microarrays measure expression levels of over 40,000 genes. A flow cytometry assay consisting of twelve markers (e.g., CD3, CD4 and HLA-DR) generates $4096\left(2^{12}\right)$ gates or possible phenotypes consisting of positive/ negative combinations of all 12 markers [3]. Research projects such as those funded in 2010 as part of the US NIH Human Immunology Project Consortium [101] are performing 51-plex Luminex, gene expression, flow phenotyping and phosphoflow assays on samples drawn from the same set of study participants at multiple time points.

For a systems perspective and for integrated biomarker discovery, we can and should combine such multiassay multianalyte data into a cohesive whole. Diabetes researchers have learned that among first-degree relatives of patients with type 1 diabetes, autoantibody positivity for two or more antibodies from a panel of three correlates with a three- to seven-fold increased risk of disease as compared with positivity for a single antibody [4]. The Cancer Vaccine Clinical Trials Working Group suggests that immune response should be measured by two assays at three time points [5]. Using multiple assays and multiple analytes can increase the credibility and predictive value of putative biomarker panels.

\section{The case for data warehousing}

Since a study design involving multiple assays is likely to involve instruments from several manufacturers and include clinical data from an institution-specific clinical database, it is unlikely that instrument manufacturers will provide software for combining heterogeneous data. Fortunately, the discipline of data warehousing offers a body of literature, and associated tools and techniques to address this challenge [6,7]. A data warehouse combines heterogeneous data in a consistent fashion to support analysis and decision making. A data warehouse has three key components: the dimensional model, the backend processes and end-user applications. Each of these components is described below:

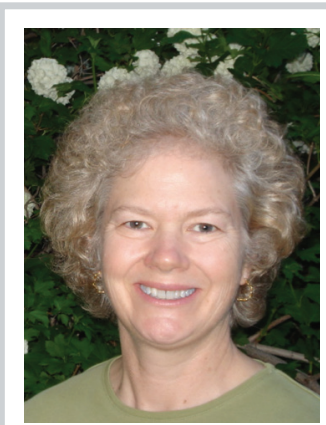

Janet Siebert

CytoAnalytics, 1080 Bonnie Brae Blvd, Denver, CO 80209, USA

Tel.: +l 3037330923

E-mail: jsiebert@cytoanalytics.com

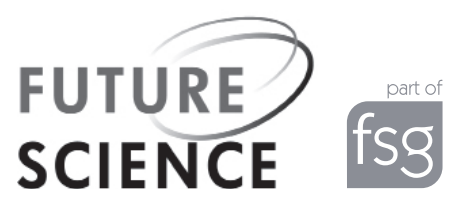


- The dimensional model is a way of organizing heterogeneous data that is easy to understand and that supports rapid retrieval of data. In a dimensional model, a numeric fact (e.g., an assay readout) is described by a variety of attributes, called dimensions. For example, in immune monitoring, some of the relevant dimensions might be assay (e.g., Luminex, flow phenotyping), analyte (e.g., IFN- $\gamma$, IL-10, $\mathrm{CD}^{+}{ }^{+} \mathrm{CD} 4{ }^{+}$regulatory $\mathrm{T}$ cells), units of measure (e.g., $\mathrm{pg} / \mathrm{ml}$, frequency of parent population, fold change), tissue (e.g., serum, peripheral blood mononuclear cells), participant condition (e.g., case, control), and sample timepoint (e.g., pretreatment, posttreatment). The dimensional model is often implemented in a relational database;

- The backend processes extract data from source systems and then transform and load these data into the dimensional model. Collectively, these processes are known as extract, transform, load (ETL). In immune monitoring, the extracts are often provided by software that runs on the analytical instrument itself or by downstream software that provides some level of feature extraction, such as curve fitting in the case of Luminex assays, gating of events in the case of flow cytometry assays and image quantification in the case of microarrays. These extracts can include results for just one sample or for a batch of samples (e.g., a plate), depending on the instrument and associated software;

- End-user applications support data exploration and decision-making. In the case of life science research, these applications should also support custom data extracts that in turn are input to sophisticated bioinformatics or statistical analysis.

The process of building dimensional databases is not innovative computer science, but rather the implementation of well-established practices requiring specialized skills. Successful practitioners understand complex datasets in multiple formats, such as those generated by many different instruments, and in the consolidated dimensional model of the data warehouse. These practitioners can conceptualize how the data must be transformed from its original format into the dimensional model. They also have the skills to program the transformations. Just as not all biologists are experts in tumor immunology, not all computer scientists are experts in combining heterogeneous data. In addition, since the discipline of data warehousing is well established, it does not offer attractive research opportunities for computer scientists who are expected to publish innovative contributions to their field. One might describe data warehousing as a computer science analogue of the vivarium. Consequently, data warehousing expertise is generally more common in industry than in academia. Therefore, biologists interested in pursuing integrated biomarker discovery in an academic research setting are faced with the challenge of finding computer scientists with expertise in and enthusiasm for data warehousing.

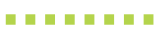

"Using multiple assays and multiple analytes can increase the credibility and predictive value of putative biomarker panels."

ma mam ma

Given this challenge, we might wonder whether combining data is a necessary evil or an exciting opportunity. For computer scientists and biologists who are comfortable thinking about and interacting with large datasets, a cross-assay database is truly exciting and empowering. It offers an unparalleled opportunity for integrated biomarker discovery. Designing the data transformations from original format to dimensional model is like solving a puzzle, offering a certain intellectual challenge and satisfaction to the builders. Furthermore, when built with a dimensional model, the database provides a foundation for a common cross-assay vocabulary to describe and discuss experimental results. In addition, the data can be explored and interrogated with a common cross-assay tool kit. We do not need to build or to learn specialized software tools for each platform or assay. Importantly, building the data warehouse requires collaboration among computer scientists and biologists with the right skill sets. If successful, the development process will result in a highperforming team with a shared vocabulary and the interdisciplinary communication skills to fully exploit the combined data. Thus, these factors suggest that combining heterogeneous data in a data warehouse can transform biomarker discovery.

Once the data warehouse exists, the data is available for exploration and analysis by biologists, computer scientists and statisticians. One end-user application that supports investigator-driven exploration is 
Jaspersoft, an open source business intelligence suite. This suite includes online analytical processing (OLAP). OLAP allows users to create data tables with various dimensions (e.g., treatment, response, assay, analyte) on rows or columns, and to then drill down into the data and through to the underlying detail. Similar functionality is provided by commercial tools such as Spotfire and Tableau. Such tools allow users to make hypotheses about the data and test these hypotheses within minutes. Inspection of the data often leads to other hypotheses that can be quickly explored. This interaction helps researchers become comfortable with multidimensional data and provides a foundation for multidimensional thinking [8]. Of note, exploring multidimensional data is a general need, faced by users in many domains. By building a data warehouse, we are able to leverage mature software tools from the business intelligence space for data exploration. We do not need tools specifically designed for life sciences.

In addition, combined data can be analyzed using readily available machine learning algorithms. However, the numeric ranges and magnitudes of cross-platform data vary widely. For example, serum cytokine levels as measured by Luminex might range from 1.04 to $2.61 \mathrm{pg} / \mathrm{ml}$ for IL-2, and from 9.0 to $287.0 \mathrm{pg} / \mathrm{ml}$ for IP-10 (CXCL10) [9]. Foldchange of phosphorylated protein expression between stimulated and unstimulated aliquots, as measured by a phospho-flow assay, might range from 0.25 to 8.47 [MAECKER, DAVIS, 2010 Unpubished Data]. To address these differences, cross-assay data can be normalized prior to analysis or can be analyzed with magnitudeinsensitive techniques such as decision trees or association rules. As an example, a decision tree algorithm can distinguish between responders and nonresponders by systematically selecting numeric values of analytes that provide the best classification of samples into responder and nonresponder. The final decision tree may well include multiple analytes spanning multiple assays. One advantage of using magnitude-insensitive techniques is that the assumptions made and variability introduced by normalization are avoided.

\section{The feasibility of data warehousing}

Three trends of the last decade increase the feasibility of creating data warehouses for experimental results in a reasonable timeframe and at a reasonable cost. These trends are the emergence of a commodity market for cloud servers, the increasing availability of open source software, and the evolution of the 'Software as a Service' (SaaS) business model:

- In the commodity market for cloud servers, the hardware required to support data warehousing can be procured quickly and cheaply. Vendors such as Amazon and Rackspace provide virtual private servers on good-quality hardware in professionally managed data centers. These servers can be rented for US $\$ 40$ to 700 per month, and can be procured in minutes;

- The increasing availability of open source software means that key technologies of the data warehouse architecture are available without licensing costs. These technologies include databases (e.g., MySQL, PostgreSQL), business intelligence suites (e.g., Jaspersoft, Pentaho), data mining tools (e.g., Weka) and statistical tools (e.g., R);

- The SaaS business model further supports rapid deployment and decreased development costs. In this approach, a service company rents the hardware and software to customers. Supported applications might include a project management system (e.g., Basecamp) or a data warehousing environment. The service company may also provide consulting services to support implementation and ongoing utilization. Because the service company is supporting multiple customers, an individual customer can, in theory, access expertise and high-quality support without having to engage a full-time resource.

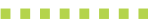 \\ "Exploring multidimensional data is a general need, faced by users in many domains. By building a data warehouse, we are able to leverage mature software tools from the business intelligence space for data exploration. We do not need tools specifically designed for life sciences."}

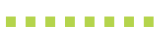

A final factor essential for deploying a data warehouse in a reasonable timeframe and at a reasonable cost is the presence of experienced team members. Skilled data warehousing professionals with a track record of success dramatically increase project feasibility. Ideally, these professionals would also have life science domain knowledge. Taken together, cloud servers, 
open source software, SaaS and experienced team members create an environment in which a data warehouse can be deployed and supported for a fraction of reagent costs.

\section{Future perspective}

In conclusion, the most valuable biomarkers may well be integrated sets, best discovered through the systematic application of multianalyte assays from a variety of instrument platforms, spanning several levels of biological processes. To thoroughly explore and interrogate the resulting data, we can leverage well-established techniques of data warehousing, combining heterogeneous data in an appropriate dimensional model. This approach offers four key advantages. First, we are able to leverage general-purpose tools and techniques to study our data. Such tools are less expensive or more mature than those designed for a limited user base. Second, we gain a common vocabulary for discussing assay results from a variety of platforms. Third, we can apply the same set of analytical tools to explore both combined data and data from different platforms. Fourth, we obtain a more complete view of the biological systems by combining data from multiple assays across multiple levels of biological process. Consequently, combining heterogeneous data into a cohesive whole is both compelling and transformative.

\section{Acknowledgments}

The author thanks Holden T Maecker and Mark M Davis of Stanford University for valuable conversations, and for the privilege of working with their complex multianalyte, multiplatform datasets; and Edwin B Walker, of the Earle A Chiles Research Institute - Providence Portland Medical Center, and Wes Munsil of CytoAnalytics for comments and insights.

\section{Financial \& competing interests disclosure}

Janet Siebert is President and Founder of CytoAnalytics, a firm specializing in the integration and analysis of life science data. The author has no other relevant affiliations or financial involvement with any organization or entity with a financial interest in or financial conflict with the subject matter or materials discussed in the manuscript apart from those disclosed.

No writing assistance was utilized in the production of this manuscript.

\section{Bibliography}

1 Kalos M. An integrative paradigm to impart quality to correlative science. J. Transl. Med. 8, 26 (2010).

2 Atkinson AJ, Colburn WA, DeGruttola VG et al. Biomarkers and surrogate endpoints: Preferred definitions and conceptual framework. Clin. Pharmacol. Ther. 69, 89-95 (2001).

3 Autissier P, Soulas C, Burdo TH et al. Evaluation of a 12-color flow cytometry panel to study lymphocyte, monocyte, and dendritic cell subsets in humans. Cytometry $A 77$, 410-419 (2010).
4 Verge CF, Gianani R, Kawasaki E, et al. Number of autoantibodies (against insulin, GAD or ICA512/IA2) rather than particular autoantibody specificities determines risk of Type 1 diabetes. J. Autoimmun. 9, 379-383 (1996).

5 Hoos A, Parmiani G, Hege K, et al. A clinical development paradigm for cancer vaccines and related biologics. J. Immunother. 30, 1-15 (2007)

6 Kimball R, Ross M, Thornthwaite W et al. The Data Warehouse Lifecycle Toolkit (2nd Edition). Wiley (2008).

7 Siebert J, Cios KJ, Newell MK. A rich analytical environment for flow cytometry experimental results. Int. J. Bioinform. Res. Appl. 2, 52-62 (2006).

8 Siebert JC, Walker EB. Monitoring cytokine profiles during immunotherapy. Immunother. 2, 799-816 (2010).

9 Dehqanzada ZA, Storrer CE, Hueman MT et al. Assessing serum cytokine profiles in breast cancer patients receiving a HER2/neu vaccine using Luminex technology. Oncol. Rep. 17, 687-694 (2007).

- Website

101 US NIH Human Immunology Project Consortium. www.immuneprofiling.org 